\title{
Utility of Galilean Symmetry in Light-Front Perturbation Theory: A Nontrivial Example in QCD
}

\author{
A. Harindranath and Rajen Kundu \\ Saha Institute of Nuclear Physics \\ Sector I, Block AF, Bidhan Nagar, Calcutta 700064 India
}

(February 6, 1998)

\begin{abstract}
Investigations have revealed a very complex structure for the coefficient functions accompanying the divergences for individual time $\left(x^{+}\right)$ordered diagrams in light-front perturbation theory. No guidelines seem to be available to look for possible mistakes in the structure of these coefficient functions emerging at the end of a long and tedious calculation, in contrast to covariant field theory. Since, in light-front field theory, transverse boost generator is a kinematical operator which acts just as the two-dimensional Galilean boost generator in non-relativistic dynamics, it may provide some constraint on the resulting structures. In this work we investigate the utility of Galilean symmetry beyond tree level in the context of coupling constant renormalization in light-front QCD using the two-component formalism. We show that for each $x^{+}$ordered diagram separately, underlying transverse boost symmetry fixes relative signs of terms in the coefficient functions accompanying the diverging logarithms. We also summarize the results leading to coupling constant renormalization for the most general kinematics.
\end{abstract}




\section{INTRODUCTION}

In light-front field theory, at present, many higher order calculations need to be performed using time $\left(x^{+}\right)$ordered perturbative techniques in order to overcome several conceptual and practical problems [1,2]. Investigations have revealed a very complex structure for the coefficient functions (accompanying the divergences) which emerge at the end of notoriously long and tedious calculations of individual time $\left(x^{+}\right)$ordered diagrams. It appears that almost no guidance is available to look for possible mistakes in these structures. In contrast, in covariant field theory, the structures accompanying the divergences are quite simple. The complexity of the former is due to the fact that power counting is different on the light-front [1]. In the latter case, simplicity of the structure is due to the underlying Lorentz symmetry (rotational and boost invariance) which can be maintained at every stage of the calculation. Since the light-front formalism do possess some kinematical symmetries, it is worthwhile to investigate whether they can provide some constraint on the possible structure of coefficient functions for individual $x^{+}$ordered diagrams.

Two of the most important kinematic symmetries in light-front field theory which are relevant especially for phenomenological concerns are the longitudinal and transverse boost symmetries. The longitudinal boost symmetry is a scale symmetry on the light-front whereas transverse boost symmetry is simply Galilean symmetry in two dimensions in non-relativistic dynamics [3]. The implications of the Galilean symmetry for the structure of the interaction vertices resulting from the light-front Hamiltonian at tree level are known. For example, the symmetry can be utilized [4] to reduce the number of free parameters in an interaction Hamiltonian constructed at tree level purely from light-front power counting. The implications of this symmetry beyond tree level is not well-understood.

In this work we investigate the question in the context of coupling constant renormalization in light-front QCD using two-component formalism [5, 6], whether and how the Galilean symmetry manifests itself beyond tree level and whether the symmetry can provide some guidance in understanding the complex structure of coefficient functions accompanying the divergences. Specifically, we study the corrections to the canonical quark-gluon vertex arising from quark-gluon and three-gluon vertices. It turns out that the accompanying structures are either proportional to the canonical vertex or independent of the total momentum momenta and thereby exhibiting transverse boost invariance. Incidentally, these processes are relevant for the calculation of asymptotic freedom in light-front QCD and we also present the $\beta$-function calculation for the most general kinematics in the two-component formalism. However, our motivation for studying these processes is quite different in the present case. We want to stress the role played by Galilean boost symmetry in ensuring the correctness of the structure of coefficient functions appearing in the calculation beyond tree level for each $x^{+}$ordered diagram separately. Such nontrivial checks are extremely helpful, for example, in extending the calculations of structure functions presented in Ref.( [7]) to the next higher order. For completeness, we mention that previous calculation [8] of vertex corrections have employed the four-component representation of Brodsky and Lepage [9] and only the final answers after summing different time orderings have been presented. A calculation [10] of the vertex correction using the two-component representation have studied only the two specific cases of helicity-flip part of the vertex (proportional to quark mass) and zero momentum $\left(q^{+, \perp}=0\right)$ limit for the gluon. For the investigation of Galilean symmetry of 
the vertex beyond tree level we need to study each time-ordered contribution separately for arbitrary momenta for the external legs, which we undertake in this work.

The plan of this paper is as follows. In section 2, we discuss aspects of transverse boost symmetry in light-front dynamics. In section 3, explicit calculations are carried out for the quark-gluon vertex at the first non-trivial order. In section 4 , we confirm the $\beta$-function calculation presented in Ref.( [10]) for the most general kinematics. Finally, a summary, discussion of the results, and their possible implications are provided in section 5 .

\section{TRANSVERSE BOOST SYMMETRY: CANONICAL CONSIDERATIONS}

In light-front theory, the generators of transverse boost are given by

$$
E^{i}=M^{+i}=\frac{1}{2} \int d x^{-} d^{2} x^{\perp}\left[x^{i} \theta^{++}-x^{+} \theta^{+i}\right]
$$

where $\theta^{\mu \nu}$ is the symmetric energy-momentum tensor. The generators $E^{i}$ leave $x^{+}=0$ invariant and hence are kinematic operators. They obey the commutation relations

$$
\left[E^{i}, P^{j}\right]=-i \delta^{i j} P^{+},\left[E^{i}, P^{-}\right]=-2 i P^{-},\left[J^{3}, E^{i}\right]=i \epsilon^{i j} E^{j},
$$

where $P^{+}$and $P^{i}$ are the longitudinal and transverse momentum operators respectively, $P^{-}$is the Hamiltonian operator, $J^{3}$ is the helicity operator, and $\epsilon^{i j}$ the two dimensional antisymmetric tensor. Thus the generators $E^{i}$ act just like Galilean boosts in the transverse plane, familiar from non-relativistic dynamics.

In light-front theory involving fermions and gauge bosons, the interaction vertices have a nontrivial structure. Galilean symmetry implies that the interaction vertices in the theory (in momentum space) are independent of the total transverse momentum in the problem. Since the issues associated with Galilean invariance are most transparent in the two-component representation, it is most convenient to use this representation [5,6] of light-front QCD in contrast to the more familiar four-component representation [9]. In this work we follow the conventions of [6] except for the commutation relations of fermionic Fock operators which we choose as

$$
\left\{b(p, \lambda), b^{\dagger}\left(p^{\prime}, \lambda^{\prime}\right)\right\}=\left\{d(p, \lambda), d^{\dagger}\left(p^{\prime}, \lambda^{\prime}\right)\right\}=2(2 \pi)^{3} p^{+} \delta_{\lambda, \lambda^{\prime}} \delta^{3}\left(p-p^{\prime}\right) .
$$

Accordingly, in our notation, the two-component fermion field is given by

$$
\xi(x)=\sum_{\lambda} \chi_{\lambda} \int \frac{d p^{+} d^{2} p^{\perp}}{2(2 \pi)^{3} \sqrt{p^{+}}}\left[b(p, \lambda) e^{-i p \cdot x}+d^{\dagger}(p,-\lambda) e^{i p \cdot x}\right] .
$$

The canonical quark-gluon vertex in our notation is (see Fig. 1)

$$
\mathcal{V}_{1}=g T^{a} \sqrt{p_{1}^{+} p_{2}^{+}} \chi_{s_{2}}^{\dagger}\left[-2 \frac{q^{\perp}}{q^{+}}+\sigma^{\perp} \frac{\sigma^{\perp} \cdot p_{1}^{\perp}}{p_{1}^{+}}+\frac{\sigma^{\perp} \cdot p_{2}^{\perp}}{p_{2}^{+}} \sigma^{\perp}+i m\left(\frac{1}{p_{1}^{+}}-\frac{1}{p_{2}^{+}}\right)\right] \chi_{s_{1}} \cdot\left(\epsilon_{\lambda}^{\perp}\right)^{*} \text {. }
$$

Since the mass term (helicity-flip interaction) is irrelevant for the Galilean invariance, we drop it in the following. Note that the vertices and energy denominators in $x^{+}$ordered diagrams in the two-component representation are manifestly invariant under Galilean boost. (See the appendix for an explicit example at one loop level). 


\section{ONE LOOP CALCULATIONS}

In the massless limit, the helicity-flip contribution vanishes and the canonical vertex has the structure

$$
\mathcal{V}_{1}=g T^{a} \sqrt{p_{1}^{+} p_{2}^{+}} \chi_{s_{2}}^{\dagger}\left[-2 \frac{q^{\perp}}{q^{+}}+\sigma^{\perp} \frac{\sigma^{\perp} \cdot p_{1}^{\perp}}{p_{1}^{+}}+\frac{\sigma^{\perp} \cdot p_{2}^{\perp}}{p_{2}^{+}} \sigma^{\perp}\right] \chi_{s_{1}} \cdot\left(\epsilon_{\lambda}^{\perp}\right)^{*} .
$$

In this section we consider corrections to this vertex at one loop level in LF Hamiltonian perturbation theory. Specifically we consider the corrections arising from quark-gluon vertex and the three-gluon vertex. Note that the corrections arising from instantaneous vertices in the theory do not contribute to the divergent structure of the vertex for zero quark mass at one loop level.

In order to perform the calculations beyond tree level, we need to regulate the loop momenta. How to introduce regulators in light-front theory is, at present, an active subject of research 111. One may (1) introduce cutoffs on the sum of light-front energies (the socalled boost invariant cut-off), or (2) choose to cutoff energy differences at vertices (which emerge naturally in similarity renormalization perturbation theory) or (3) simply cutoff single particle momenta. We employ the third choice for the regulators, namely, $k_{i}^{+}>\epsilon, \mu<$ $k_{i}^{\perp}<\Lambda$, which is simple to implement but obviously violate both longitudinal and transverse boost invariance. Since the vertices and energy denominators are explicitly invariant under the Galilean boost (see the appendix), the violation of the symmetry can occur only through the explicit appearance of total transverse momentum $P^{\perp}$ in the limits of integration. From power counting, the vertex corrections at one loop level are only logarithmically divergent in the transverse plane. Hence we expect the coefficient functions accompanying the logarithms to still exhibit the symmetry.

Consider the one loop corrections to the vertex involving two quark-gluon vertices. There are two time-ordering contributions shown in Figs. 2(a) and 2(b). The contribution from Fig. 2(a) is

$$
\begin{array}{r}
\mathcal{V}_{2 a}=\frac{g^{3}}{2(2 \pi)^{3}} T^{b} T^{a} T^{b} \sqrt{p_{1}^{+} p_{2}^{+}} \int_{\epsilon}^{p_{2}^{+}-\epsilon} d k^{+} \int d^{2} k^{\perp} \theta\left(\Lambda-\left|k^{\perp}\right|\right) \\
\frac{1}{k_{3}^{+}} \frac{1}{p_{1}^{-}-k_{1}^{-}-k_{3}^{-}} \frac{1}{p_{1}^{-}-q^{-}-k^{-}-k_{3}^{-}} \\
\sum_{\sigma_{1} \sigma_{2} \lambda_{1}} \chi_{s_{2}}^{\dagger}\left[-2 \frac{k_{3}^{\perp}}{k_{3}^{+}}+\sigma^{\perp} \frac{\sigma^{\perp} \cdot k^{\perp}}{k^{+}}+\frac{\sigma^{\perp} \cdot p_{2}^{\perp}}{p_{2}^{+}} \sigma^{\perp}\right] \chi_{\sigma_{1}} \cdot \epsilon_{\lambda_{1}}^{\perp} \\
\chi_{\sigma_{1}}^{\dagger}\left[-2 \frac{q^{\perp}}{q^{+}}+\sigma^{\perp} \frac{\sigma^{\perp} \cdot k_{1}^{\perp}}{k_{1}^{+}}+\frac{\sigma^{\perp} \cdot k^{\perp}}{k^{+}} \sigma^{\perp}\right] \chi_{\sigma_{2}} \cdot\left(\epsilon_{\lambda}^{\perp}\right)^{*} \\
\chi_{\sigma_{2}}^{\dagger}\left[-2 \frac{k_{3}^{\perp}}{k_{3}^{+}}+\sigma^{\perp} \frac{\sigma^{\perp} \cdot p_{1}^{\perp}}{p_{1}^{+}}+\frac{\sigma^{\perp} \cdot k_{1}^{\perp}}{k_{1}^{+}} \sigma^{\perp}\right] \chi_{s_{1}} \cdot\left(\epsilon_{\lambda_{1}}^{\perp}\right)^{*} .
\end{array}
$$

Here $k_{1}^{(+, \perp)}=q^{(+, \perp)}+k^{(+, \perp)}$ and $k_{3}^{(+, \perp)}=p_{2}^{(+, \perp)}-k^{(+, \perp)}$.

After a long and tedious calculation, we arrive at two types of divergent contributions, one containing product of logarithms and other containing a single logarithm. We have taken $\mu$ and $\Lambda$ to be much larger than the external momentum scales in the problem. Divergent contributions that contain products of logarithms: 


$$
\begin{gathered}
\mathcal{V}_{2 a}^{I}=g T^{a} \sqrt{p_{1}^{+} p_{2}^{+}} \chi_{s_{2}}^{\dagger}\left[-2 \frac{q^{\perp}}{q^{+}}+\sigma^{\perp} \frac{\sigma^{\perp} \cdot p_{1}^{\perp}}{p_{1}^{+}}+\frac{\sigma^{\perp} \cdot p_{2}^{\perp}}{p_{2}^{+}} \sigma^{\perp}\right] \chi_{s_{1}} \cdot\left(\epsilon_{\lambda}^{\perp}\right)^{*} \\
\frac{g^{2}}{8 \pi^{2}}\left(-\frac{1}{2} C_{A}+C_{f}\right) \ln \frac{\Lambda}{\mu} 4 \ln \frac{p_{2}^{+}}{\epsilon}
\end{gathered}
$$

Since the coefficient of the divergent factor is proportional to the canonical vertex, the transverse boost invariance of the above result is manifest. Divergent contributions that contain single logarithm are

$$
\begin{gathered}
\mathcal{V}_{2 a}^{I I}=g T^{a} \sqrt{p_{1}^{+} p_{2}^{+}} \chi_{s_{2}}^{\dagger}\left[6 \frac{q^{\perp}}{q^{+}}-6 \frac{p_{1}^{\perp}}{p_{1}^{+}}-\frac{\sigma^{\perp} \cdot p_{2}^{\perp}}{p_{1}^{+}} \sigma^{\perp}+\frac{p_{2}^{+}}{p_{1}^{+}} \frac{\sigma^{\perp} \cdot p_{1}^{\perp}}{p_{1}^{+}} \sigma^{\perp}\right] \chi_{s_{1}} \cdot\left(\epsilon_{\lambda}^{\perp}\right)^{*} \\
\frac{g^{2}}{8 \pi^{2}}\left(-\frac{1}{2} C_{A}+C_{f}\right) \ln \frac{\Lambda}{\mu} .
\end{gathered}
$$

In this case the coefficient of the divergent factor involving transverse momenta is not proportional to the canonical vertex. However, in terms of the internal momenta (see the appendix), the quantity inside the square bracket can be rewritten as

$$
\begin{aligned}
& {\left[6 \frac{q^{\perp}}{q^{+}}-6 \frac{p_{1}^{\perp}}{p_{1}^{+}}-\frac{\sigma^{\perp} \cdot p_{2}^{\perp}}{p_{1}^{+}} \sigma^{\perp}+\frac{p_{2}^{+}}{p_{1}^{+}} \frac{\sigma^{\perp} \cdot p_{1}^{\perp}}{p_{1}^{+}} \sigma^{\perp}\right]} \\
& =-\frac{1}{P^{+}}\left[\frac{6 \kappa_{1}^{\perp}}{1-x}+\sigma^{\perp} . \kappa_{1}^{\perp} \sigma^{\perp}\right],
\end{aligned}
$$

which satisfies the constraint from Galilean invariance.

Contribution from Fig. 2(b) is

$$
\begin{gathered}
\mathcal{V}_{2 b}=(-) \frac{g^{3}}{2(2 \pi)^{3}} T^{b} T^{a} T^{b} \sqrt{p_{1}^{+} p_{2}^{+}} \int_{\epsilon}^{q^{+}-\epsilon} d k_{2}^{+} \int d^{2} k_{2}^{\perp} \theta\left(\Lambda-\left|k^{\perp}\right|\right) \frac{1}{p_{1}^{+}-k_{2}^{+}} \frac{1}{p_{1}^{-}-k_{1}^{-}-k_{2}^{-}} \\
\frac{1}{p_{1}^{-}-k_{2}^{-}-k_{3}^{-}-p_{2}^{-}} \sum_{\sigma_{1} \sigma_{2} \lambda_{1}} \chi_{s_{2}}^{\dagger}\left[-2 \frac{k_{1}^{\perp}}{k_{1}^{+}}+\sigma^{\perp} \frac{\sigma^{\perp} \cdot k_{3}^{\perp}}{k_{3}^{+}}+\frac{\sigma^{\perp} \cdot p_{2}^{\perp}}{p_{2}^{+}} \sigma^{\perp}\right] \chi_{\sigma_{1}} \cdot \epsilon_{\lambda_{1}}^{\perp} \\
\chi_{\sigma_{1}}^{\dagger}\left[-2 \frac{q^{\perp}}{q^{+}}+\sigma^{\perp} \frac{\sigma^{\perp} \cdot k_{2}^{\perp}}{k_{2}^{+}}+\frac{\sigma^{\perp} \cdot k_{3}^{\perp}}{k_{3}^{+}} \sigma^{\perp}\right] \chi_{\sigma_{2}} \cdot\left(\epsilon_{\lambda}^{\perp}\right)^{*} \\
\chi_{\sigma_{2}}^{\dagger}\left[-2 \frac{k_{1}^{\perp}}{k_{1}^{+}}+\sigma^{\perp} \frac{\sigma^{\perp} \cdot p_{1}^{\perp}}{p_{1}^{+}}+\frac{\sigma^{\perp} \cdot k_{2}^{\perp}}{k_{2}^{+}} \sigma^{\perp}\right] \chi_{s_{1}} \cdot\left(\epsilon_{\lambda_{1}}^{\perp}\right)^{*} .
\end{gathered}
$$

Here $k_{1}^{(+, \perp)}=p_{1}^{(+, \perp)}-k_{2}^{(+, \perp)}$ and $k_{3}^{(+, \perp)}=q^{(+, \perp)}-k_{2}^{(+, \perp)}$. The overall -ve sign arises from the anti symmetry property of fermionic states. Note that this -ve sign is missing from Eq. (A8) of Ref. [10].

As in the previous case, explicit evaluation leads to terms containing two types of divergences. Divergent contributions that contain products of logarithms are

$$
\begin{gathered}
\mathcal{V}_{2 b}^{I}=g T^{a} \sqrt{p_{1}^{+}} \sqrt{p_{2}^{+}} \chi_{s_{2}}^{\dagger}\left[-2 \frac{q^{\perp}}{q^{+}}+\sigma^{\perp} \frac{\sigma^{\perp} \cdot p_{1}^{\perp}}{p_{1}^{+}}+\frac{\sigma^{\perp} \cdot p_{2}^{\perp}}{p_{2}^{+}} \sigma^{\perp}\right] \chi_{s_{1}} \cdot\left(\epsilon_{\lambda}^{\perp}\right)^{*} \\
\frac{g^{2}}{8 \pi^{2}}\left(-\frac{1}{2} C_{A}+C_{f}\right) \ln \frac{\Lambda}{\mu} 2 \ln \frac{p_{1}^{+}}{p_{2}^{+}} .
\end{gathered}
$$


Again, the transverse boost invariance of this result is manifest since the contribution is proportional to the canonical vertex. Divergent contributions that contain single logarithm are

$$
\begin{aligned}
\mathcal{V}_{2 b}^{I I}= & (-) g T^{a} \sqrt{p_{1}^{+} p_{2}^{+}} \chi_{s_{2}}^{\dagger}\left[3 \sigma^{\perp} \frac{\sigma^{\perp} \cdot p_{1}^{\perp}}{p_{1}^{+}}+3 \frac{\sigma^{\perp} \cdot p_{2}^{\perp}}{p_{2}^{+}} \sigma^{\perp}\right. \\
& \left.-6 \frac{p_{1}^{\perp}}{p_{1}^{+}}-\frac{\sigma^{\perp} \cdot p_{2}^{\perp}}{p_{1}^{+}} \sigma^{\perp}+\frac{p_{2}^{+}}{p_{1}^{+}} \frac{\sigma^{\perp} \cdot p_{1}^{\perp}}{p_{1}^{+}} \sigma^{\perp}\right] \chi_{s_{1}} \cdot\left(\epsilon_{\lambda}^{\perp}\right)^{*} \\
& \frac{g^{2}}{8 \pi^{2}}\left(-\frac{1}{2} C_{A}+C_{f}\right) \ln \frac{\Lambda}{\mu} .
\end{aligned}
$$

The transverse boost symmetry of the terms inside the square bracket is not manifest but becomes explicit once we express the result in terms of the internal momenta. Alternatively, by subtracting and adding the term $-6 \frac{q^{\perp}}{q^{+}}$to these terms we can rewrite the terms inside the square bracket as the canonical term plus the terms contained in the square bracket in eq.(3.4) which again shows the boost invariance of the result in eq.(3.8).

Consider, next, the one loop contributions to the quark-gluon vertex involving one quarkgluon vertex and one three gluon vertex. There are two time ordering contributions shown in Figs. 3(a) and 3(b). The contribution from Fig. 3(a) is

$$
\begin{gathered}
\mathcal{V}_{3 a}=\frac{g^{3}}{2(2 \pi)^{3}}\left(-i f^{a b c} T^{b} T^{c}\right) \sqrt{p_{1}^{+} p_{2}^{+}} \int_{\epsilon}^{p_{2}^{+}-\epsilon} d k^{+} \int d^{2} k^{\perp} \theta\left(\Lambda-\left|k^{\perp}\right|\right) \frac{1}{k_{1}^{+}} \frac{1}{k_{2}^{+}} \frac{1}{p_{1}^{-}-k_{1}^{-}-k^{-}} \\
\frac{1}{p_{1}^{-}-q^{-}-k_{2}^{-}-k^{-}} \sum_{\sigma_{1}, \lambda_{1}, \lambda_{2}} \chi_{s_{2}}^{\dagger}\left[-2 \frac{k_{2}^{\perp}}{k_{2}^{+}}+\sigma^{\perp} \frac{\sigma^{\perp} \cdot k^{\perp}}{k^{+}}+\frac{\sigma^{\perp} \cdot p_{2}^{\perp}}{p_{2}^{+}} \sigma^{\perp}\right] \chi_{\sigma_{1}} \cdot \epsilon_{\lambda_{2}}^{\perp} \\
\chi_{\sigma_{1}}^{\dagger}\left[-2 \frac{k_{1}^{\perp}}{k_{1}^{+}}+\sigma^{\perp} \frac{\sigma^{\perp} \cdot p_{1}^{\perp}}{p_{1}^{+}}+\frac{\sigma^{\perp} \cdot k^{\perp}}{k^{+}} \sigma^{\perp}\right] \chi_{s_{1}} \cdot\left(\epsilon_{\lambda_{1}}^{\perp}\right)^{*} \\
\epsilon_{\lambda_{1}}^{j}\left(\epsilon_{\lambda}^{i}\right)^{*}\left(\epsilon_{\lambda_{2}}^{l}\right)^{*}\left[\left[\left(k_{1}^{i}+k_{2}^{i}\right)-\frac{q^{i}}{q^{+}}\left(k_{1}^{+}+k_{2}^{+}\right)\right] \delta_{l j}-\left[\left(k_{1}^{l}+q^{l}\right)-\frac{k_{2}^{l}}{k_{2}^{+}}\left(k_{1}^{+}+q^{+}\right)\right] \delta_{i j}\right. \\
\left.+\left[\left(q^{j}-k_{2}^{j}\right)-\frac{k_{1}^{j}}{k_{1}^{+}}\left(q^{+}-k_{2}^{+}\right)\right] \delta_{i l}\right] .
\end{gathered}
$$

Here $k_{1}^{(+, \perp)}=p_{1}^{(+, \perp)}-k^{(+, \perp)}$ and $k_{2}^{(+, \perp)}=p_{2}^{(+, \perp)}-k^{(+, \perp)}$. Divergent contributions that contain products of logarithms are

$$
\begin{gathered}
\mathcal{V}_{3 a}^{I}=g T^{a} \sqrt{p_{1}^{+} p_{2}^{+}} \chi_{s_{2}}^{\dagger}\left[-2 \frac{q^{\perp}}{q^{+}}+\sigma^{\perp} \frac{\sigma^{\perp} \cdot p_{1}^{\perp}}{p_{1}^{+}}+\frac{\sigma^{\perp} \cdot p_{2}^{\perp}}{p_{2}^{+}} \sigma^{\perp}\right] \chi_{s_{1}} \cdot\left(\epsilon_{\lambda}^{\perp}\right)^{*} \\
\frac{g^{2}}{8 \pi^{2}} \frac{1}{2} C_{A} \ln \frac{\Lambda}{\mu} 2 \ln \frac{p_{1}^{+} p_{2}^{+}}{q^{+} \epsilon} .
\end{gathered}
$$

The boost invariance of this result is again clear. Divergent contributions that contain single logarithm are

$$
\begin{gathered}
\mathcal{V}_{3 a}^{I I}=g T^{a} \sqrt{p_{1}^{+} p_{2}^{+}} \chi_{s_{2}}^{\dagger}\left[6 \frac{q^{\perp}}{q^{+}}-6 \frac{p_{1}^{\perp}}{p_{1}^{+}}+\frac{\sigma^{\perp} \cdot p_{2}^{\perp}}{p_{1}^{+}} \sigma^{\perp}-\frac{p_{2}^{+}}{p_{1}^{+}} \frac{\sigma^{\perp} \cdot p_{1}^{\perp}}{p_{1}^{+}} \sigma^{\perp}\right] \chi_{s_{1}} \cdot\left(\epsilon_{\lambda}^{\perp}\right)^{*} \\
\frac{g^{2}}{8 \pi^{2}} \frac{1}{2} C_{A} \ln \frac{\Lambda}{\mu} .
\end{gathered}
$$


Expressing the terms inside the square bracket in terms of the internal momenta we get $-\frac{1}{P^{+}}\left[\frac{6 \kappa_{1}^{\perp}}{1-x}-\sigma^{\perp} \cdot \kappa_{1}^{\perp} \sigma^{\perp}\right]$ which makes boost invariance explicit.

The contribution from Fig. 3(b) is

$$
\begin{gathered}
\mathcal{V}_{3 b}=\frac{g^{3}}{2(2 \pi)^{3}}\left(-i f^{a b c} T^{b} T^{c}\right) \sqrt{p_{1}^{+} p_{2}^{+}} \int_{\epsilon}^{q^{+}-\epsilon} d k_{1}^{+} \int d^{2} k_{1}^{\perp} \theta\left(\Lambda-\left|k^{\perp}\right|\right) \frac{1}{k_{1}^{+}} \frac{1}{k_{2}^{+}} \frac{1}{p_{1}^{-}-k_{1}^{-}-k^{-}} \\
\frac{1}{p_{1}^{-}-k_{1}^{-}-k_{2}^{-}-p_{2}^{-}} \sum_{\sigma_{1} \lambda_{1} \lambda_{2}} \chi_{s_{2}}^{\dagger}\left[-2 \frac{k_{2}^{\perp}}{k_{2}^{+}}+\sigma^{\perp} \frac{\sigma^{\perp} \cdot k^{\perp}}{k^{+}}+\frac{\sigma^{\perp} \cdot p_{2}^{\perp}}{p_{2}^{+}} \sigma^{\perp}\right] \chi_{\sigma_{1}} \cdot\left(\epsilon_{\lambda_{2}}^{\perp}\right)^{*} \\
\chi_{\sigma_{1}}^{\dagger}\left[-2 \frac{k_{1}^{\perp}}{k_{1}^{+}}+\sigma^{\perp} \frac{\sigma^{\perp} \cdot p_{1}^{\perp}}{p_{1}^{+}}+\frac{\sigma^{\perp} \cdot k^{\perp}}{k^{+}} \sigma^{\perp}\right] \chi_{s_{1}} \cdot\left(\epsilon_{\lambda_{1}}^{\perp}\right)^{*} \\
\epsilon_{\lambda_{1}}^{j}\left(\epsilon_{\lambda}^{i}\right)^{*} \epsilon_{\lambda_{2}}^{l}\left[\left[\left(k_{1}^{i}-k_{2}^{i}\right)-\frac{q^{i}}{q^{+}}\left(k_{1}^{+}-k_{2}^{+}\right)\right] \delta_{l j}-\left[\left(k_{1}^{l}+q^{l}\right)-\frac{k_{2}^{l}}{k_{2}^{+}}\left(k_{1}^{+}+q^{+}\right)\right] \delta_{i j}\right. \\
\left.+\left[\left(q^{j}+k_{2}^{j}\right)-\frac{k_{1}^{j}}{k_{1}^{+}}\left(q^{+}+k_{2}^{+}\right)\right] \delta_{i l}\right] .
\end{gathered}
$$

Here $k^{(+, \perp)}=p_{1}^{(+, \perp)}-k_{1}^{(+, \perp)}$ and $k_{2}^{(+, \perp)}=q^{(+, \perp)}-k_{1}^{(+, \perp)}$.

Divergent contributions that contain products of logarithms are

$$
\begin{gathered}
\mathcal{V}_{3 b}^{I}=g T^{a} \sqrt{p_{1}^{+} p_{2}^{+}} \chi_{s_{2}}^{\dagger}\left[-2 \frac{q^{\perp}}{q^{+}}+\sigma^{\perp} \frac{\sigma^{\perp} \cdot p_{1}^{\perp}}{p_{1}^{+}}+\frac{\sigma^{\perp} \cdot p_{2}^{\perp}}{p_{2}^{+}} \sigma^{\perp}\right] \chi_{s_{1}} \cdot\left(\epsilon_{\lambda}^{\perp}\right)^{*} \\
\frac{g^{2}}{8 \pi^{2}} \frac{1}{2} C_{A} \ln \frac{\Lambda}{\mu} 6 \ln \frac{q^{+}}{\epsilon}
\end{gathered}
$$

which is manifestly boost invariant. Divergent contributions that contain single logarithm are

$$
\begin{aligned}
\mathcal{V}_{3 b}^{I I}=g & T^{a} \sqrt{p_{1}^{+}} \sqrt{p_{2}^{+}} \chi_{s_{2}}^{\dagger}\left[-3 \sigma^{\perp} \frac{\sigma^{\perp} \cdot p_{1}^{\perp}}{p_{1}^{+}}-3 \frac{\sigma^{\perp} \cdot p_{2}^{\perp}}{p_{2}^{+}} \sigma^{\perp}\right. \\
& \left.+6 \frac{p_{1}^{\perp}}{p_{1}^{+}}+\frac{p_{2}^{+}}{p_{1}^{+}} \frac{\sigma^{\perp} \cdot p_{1}^{\perp}}{p_{1}^{+}} \sigma^{\perp}-\frac{\sigma^{\perp} \cdot p_{2}^{\perp}}{p_{1}^{+}} \sigma^{\perp}\right] \chi_{s_{1}} \cdot\left(\epsilon_{\lambda}^{\perp}\right)^{*} \frac{g^{2}}{8 \pi^{2}} \frac{1}{2} C_{A} \ln \frac{\Lambda}{\mu} .
\end{aligned}
$$

Comparison with eq.(3.8) again makes the boost invariance of this answer explicit.

\section{COUPLING CONSTANT RENORMALIZATION}

For the sake of completeness, we present here the results for the other diagrams which are relevant for the coupling constant renormalization. We also calculate the $\beta$-function which exactly matches with the well known results and therefore extends the results arrived at in the Ref.( [10]), to the most general kinematics in the two-component formalism.

The sum of divergent contributions from Figs. 2(a) and 2(b) is

$$
\begin{aligned}
\mathcal{V}_{2}= & g T^{a} \sqrt{p_{1}^{+} p_{2}^{+}} \chi_{s_{2}}^{\dagger}\left[-2 \frac{q^{\perp}}{q^{+}}+\sigma^{\perp} \frac{\sigma^{\perp} \cdot p_{1}^{\perp}}{p_{1}^{+}}+\frac{\sigma^{\perp} \cdot p_{2}^{\perp}}{p_{2}^{+}} \sigma^{\perp}\right] \chi_{s_{1}} \cdot\left(\epsilon_{\lambda}^{\perp}\right)^{*} \\
& \frac{g^{2}}{8 \pi^{2}}\left(-\frac{1}{2} C_{A}+C_{f}\right) \ln \frac{\Lambda}{\mu}\left(2 \ln \frac{p_{1}^{+} p_{2}^{+}}{\epsilon^{2}}-3\right),
\end{aligned}
$$


where we observe the emergence of the canonical vertex structure.

The sum of divergent contributions from Figs. 3(a) and 3(b) is

$$
\begin{gathered}
\mathcal{V}_{3}=g T^{a} \sqrt{p_{1}^{+}} \sqrt{p_{2}^{+}} \chi_{s_{2}}^{\dagger}\left[-2 \frac{q^{\perp}}{q^{+}}+\sigma^{\perp} \frac{\sigma^{\perp} \cdot p_{1}^{\perp}}{p_{1}^{+}}+\frac{\sigma^{\perp} \cdot p_{2}^{\perp}}{p_{2}^{+}} \sigma^{\perp}\right] \chi_{s_{1}} \cdot\left(\epsilon_{\lambda}^{\perp}\right)^{*} \\
\frac{g^{2}}{8 \pi^{2}} \frac{1}{2} C_{A} \ln \frac{\Lambda}{\mu}\left(2 \ln \frac{p_{1}^{+} p_{2}^{+}}{\epsilon^{2}}+4 \ln \frac{q^{+}}{\epsilon}-3\right),
\end{gathered}
$$

where we again observe the emergence of the canonical vertex.

The diagrams in Figs. 4(a), 4(b), 5(a) and 5(b) correspond to the renormalization of the external quark and gluon legs that are connected to the vertex. Their contributions are given below.

$$
\begin{aligned}
& \mathcal{V}_{4 a}=g T^{a} \sqrt{p_{1}^{+} p_{2}^{+}} \chi_{s_{2}}^{\dagger} \quad\left[-2 \frac{q^{\perp}}{q^{+}}+\sigma^{\perp} \frac{\sigma^{\perp} \cdot p_{1}^{\perp}}{p_{1}^{+}}+\frac{\sigma^{\perp} \cdot p_{2}^{\perp}}{p_{2}^{+}} \sigma^{\perp}\right] \chi_{s_{1}} \cdot\left(\epsilon_{\lambda}^{\perp}\right)^{*} \\
& \frac{g^{2}}{4 \pi^{2}} C_{f} \ln \frac{\Lambda}{\mu}\left(\frac{3}{2}-2 \ln \frac{p_{1}^{+}}{\epsilon}\right) \text {, } \\
& \mathcal{V}_{4 b}=g T^{a} \sqrt{p_{1}^{+} p_{2}^{+}} \chi_{s_{2}}^{\dagger} \quad\left[-2 \frac{q^{\perp}}{q^{+}}+\sigma^{\perp} \frac{\sigma^{\perp} \cdot p_{1}^{\perp}}{p_{1}^{+}}+\frac{\sigma^{\perp} \cdot p_{2}^{\perp}}{p_{2}^{+}} \sigma^{\perp}\right] \chi_{s_{1}} \cdot\left(\epsilon_{\lambda}^{\perp}\right)^{*} \\
& \frac{g^{2}}{4 \pi^{2}} C_{f} \ln \frac{\Lambda}{\mu}\left(\frac{3}{2}-2 \ln \frac{p_{2}^{+}}{\epsilon}\right), \\
& \mathcal{V}_{5 a}=-g T^{a} \sqrt{p_{1}^{+} p_{2}^{+}} \chi_{s_{2}}^{\dagger} \quad\left[-2 \frac{q^{\perp}}{q^{+}}+\sigma^{\perp} \frac{\sigma^{\perp} \cdot p_{1}^{\perp}}{p_{1}^{+}}+\frac{\sigma^{\perp} \cdot p_{2}^{\perp}}{p_{2}^{+}} \sigma^{\perp}\right] \chi_{s_{1}} \cdot\left(\epsilon_{\lambda}^{\perp}\right)^{*} \\
& \frac{g^{2}}{8 \pi^{2}} \frac{4}{3} N_{f} T_{f} \ln \frac{\Lambda}{\mu} \text {. } \\
& \mathcal{V}_{5 b}=g T^{a} \sqrt{p_{1}^{+} p_{2}^{+}} \chi_{s_{2}}^{\dagger} \quad\left[-2 \frac{q^{\perp}}{q^{+}}+\sigma^{\perp} \frac{\sigma^{\perp} \cdot p_{1}^{\perp}}{p_{1}^{+}}+\frac{\sigma^{\perp} \cdot p_{2}^{\perp}}{p_{2}^{+}} \sigma^{\perp}\right] \chi_{s_{1}} \cdot\left(\epsilon_{\lambda}^{\perp}\right)^{*} \\
& \frac{g^{2}}{8 \pi^{2}} C_{A} \ln \frac{\Lambda}{\mu}\left(\frac{11}{3}-4 \ln \frac{q^{+}}{\epsilon}\right),
\end{aligned}
$$

Now, to evaluate the contributions to the coupling constant, we have to multiply $\mathcal{V}_{4}$ and $\mathcal{V}_{5}$ with $\frac{1}{2}$ in order to take into account the proper correction due to the renormalization of initial and final states [12]. Thus adding the contributions we get,

$$
\begin{aligned}
\delta \mathcal{V}_{1}= & \left(\frac{1}{2} \mathcal{V}_{4}+\frac{1}{2} \mathcal{V}_{5}+\mathcal{V}_{2}+\mathcal{V}_{3}\right) \\
= & g T^{a} \sqrt{p_{1}^{+} p_{2}^{+}} \chi_{s_{2}}^{\dagger}\left[-2 \frac{q^{\perp}}{q^{+}}+\sigma^{\perp} \frac{\sigma^{\perp} \cdot p_{1}^{\perp}}{p_{1}^{+}}+\frac{\sigma^{\perp} \cdot p_{2}^{\perp}}{p_{2}^{+}} \sigma^{\perp}\right] \chi_{s_{1}} \cdot\left(\epsilon_{\lambda}^{\perp}\right)^{*} \\
& \frac{g^{2}}{8 \pi^{2}}\left(\frac{11}{6} C_{A}-\frac{2}{3} N_{f} T_{f}\right) \ln \frac{\Lambda}{\mu}
\end{aligned}
$$


Note that all the mixed divergences cancel. The correction to the coupling constant is given by

$$
g_{R}=g(1+\delta g)=g\left[1+\frac{g^{2}}{8 \pi^{2}}\left(\frac{11}{6} C_{A}-\frac{2}{3} N_{f} T_{f}\right) \ln \frac{\Lambda}{\mu}\right] .
$$

We compute the $\beta$-function as

$$
\begin{aligned}
\beta(g) & =-\frac{\partial g_{R}}{\partial \ln \Lambda} \\
& =-\frac{g^{3}}{16 \pi^{2}}\left(\frac{11}{3} C_{A}-\frac{4}{3} N_{f} T_{f}\right),
\end{aligned}
$$

which is well known result to the one-loop order.

\section{SUMMARY, DISCUSSION AND CONCLUSIONS}

Calculations employing time $\left(x^{+}\right)$ordered perturbative techniques in light-front theory are known to be straightforward but long and tedious. A lot of effort has to be invested in the calculation of coefficient functions accompanying the divergences for individual diagrams. To the best of our knowledge, this is the first work to investigate the utility of Galilean boost symmetry in determining the correctness of the structure of the coefficient functions accompanying the divergences in light-front perturbation theory beyond tree level.

In this initial investigation we have employed the simplest choice of regulators that cutoff single particle momenta. One should note that in addition to possible violations of boost invariance, such simple minded cutoff procedure could in principle even introduce non-analyticities in the structure of counterterms (see Sec. VI of Ref. [1] for an explicit example). However, in the case of vertex diagrams, we encounter only logarithmic transverse divergences. Even with finite cutoffs, violations of transverse boost invariance can appear only inside the logarithms and we expect the symmetry to be present in the non-trivial structure of the coefficient functions that accompany the divergences. We are primarily interested in understanding the complex structure of these coefficient functions on the basis of Galilean symmetry. Incidentally we note that, in contrast, longitudinal boost invariance is a scale invariance in light-front theory. The implication of longitudinal boost symmetry for the coefficient functions is trivial, namely, simple scaling behavior.

Let us summarize our findings. Out of all the $x^{+}$-ordered diagrams relevant for our calculation, four involve wavefunction renormalization correction and have the structure of the canonical vertex. For the remaining diagrams which correspond to vertex corrections, the divergent contributions from each of the them contain terms that involve (I) product of logarithms and (II) single logarithm. For contributions that belong to (I), we find that for each diagram separately, the coefficient of the divergent factor is proportional to the canonical vertex and hence Galilean boost invariance is manifestly maintained. For contributions that belong to (II), for each diagram, the coefficient of the divergent factor is not proportional to the canonical vertex. Nevertheless, in each case, rewriting the coefficient in terms of the internal momenta explicitly shows that the coefficient is independent of the 
total transverse momentum $P^{\perp}$. Hence for the contributions that belong to (II) the constraint from transverse boost invariance is maintained, even though the canonical form is not reproduced.

Our results show that two-dimensional Galilean invariance which is manifest at tree level is also exhibited in the coefficient functions accompanying the divergences in the regulated theory at the one loop level in the case of quark-gluon vertex in light-front QCD even with a regulator that violates the symmetry. Since the symmetry is only a part of the complete Lorentz symmetry, we expect the constraints which follow from the invariance to be less restrictive. Indeed, our results show that the structure of the vertex that satisfies transverse boost invariance is not unique.

Even-though the canonical vertex structure is not reproduced in the coefficient of the single logarithms, it still has some usefulness in practical calculations since it obeys constraint from Galilean boost invariance. The coefficient functions accompanying single logarithms are obtained after isolating the leading double logarithms and they exhibit a complicated structure. It is quite easy to make a mistake in the sign in one of the terms for individual $x^{+}$ordered diagrams. Our calculations show that using the underlying transverse boost symmetry one can easily recognize the mistake in the calculation and hence correct it.

We have also summarized the results for the complete set of diagrams contributing to coupling constant renormalization for the massless quark case. We have extracted the $\beta$ function which matches with the well-known results and therefore extends the results arrived previously to the most general kinematics. Using the two-component representation [6] we have presented for the first time the results separately for each $x^{+}$ordered diagram with arbitrary external momenta which is essential to study the renormalization of the helicity-non flip parts of the vertex. Present calculations together with the calculations presented in Ref. [10] explicitly show that linear divergences of the type $\frac{1}{\epsilon}$ where $\epsilon$ is the cutoff on longitudinal loop momenta occur in individual time-ordered diagrams only in radiative corrections to the chiral symmetry breaking part of the quark gluon vertex. This divergence is a special feature of non-abelian gauge theory. At one loop level, this divergence cancels with our choice of regulators when different time-ordered diagrams are summed up. Since intermediate states involved are, in general, different in different time ordered diagrams, the cancellation may no longer be operative once more sophisticated regulators that explicitly depend on the intermediate states are employed. This needs to be investigated in detail in the future because of its nontrivial consequences for the renormalization of chiral symmetry breaking terms in the QCD Hamiltonian.

The present calculations are also essential for the development of a new method [13] of calculation of structure functions in deep inelastic scattering. This approach combines the techniques of light-front current algebra and Fock space expansion for the Hamiltonian in the light-front gauge $A^{+}=0$, to treat the non-perturbative and perturbative parts of the structure functions in the same language, namely, that of multi-parton wavefunctions. Up to now, renormalization has been performed in this framework to second order for unpolarized and polarized structure functions in perturbative QCD [7]. The calculations presented in this paper constitute essential parts of a complete fourth order analysis of leading logarithms which is necessary to establish the viability of the new approach. 


\section{APPENDIX A: MANIFEST BOOST SYMMETRY OF ENERGY DENOMINATORS AND VERTICES}

In this appendix we verify the Galilean boost invariance of vertices and energy differences that occur in light-front time-ordered loop diagrams. First consider the canonical vertex given in eq.(2.5). Let $P^{+}$and $P^{\perp}$ denote total longitudinal and transverse momentum in the problem. We introduce the momentum fractions $x_{i}$ and the relative transverse momenta $\kappa_{i}^{\perp}$ by

$$
p_{2}^{+}=x P^{+}, p_{2}^{\perp}=\kappa_{1}^{\perp}+x P^{\perp}, q^{+}=(1-x) P^{+}, q^{\perp}=-\kappa_{1}^{\perp}+(1-x) P^{\perp} .
$$

The longitudinal momentum fractions $x_{i}$ and the relative transverse momenta $\kappa_{i}^{\perp}$ obey the constraints $\sum x_{i}=1$ and $\sum \kappa_{i}^{\perp}=0$. The canonical vertex takes the form

$$
\mathcal{V}_{0}=g T^{a} \sqrt{x} \chi_{s_{2}}^{\dagger}\left[2 \frac{\kappa_{1}^{\perp}}{1-x}+\frac{\sigma^{\perp} \cdot \kappa_{1}^{\perp}}{x} \sigma^{\perp}+i m\left(1-\frac{1}{x}\right)\right] \chi_{s_{1}} \cdot\left(\epsilon_{\lambda}^{\perp}\right)^{*} .
$$

In terms of the internal momenta, the boost invariance of the quark-gluon vertex is clearly manifest.

Next consider loop diagrams. As an example we consider the diagram shown in Fig. 2(a). Parameterize the single particle momenta in terms of the internal momenta as follows.

$$
k_{3}^{+}=y P^{+}, k_{3}^{\perp}=\kappa_{2}^{\perp}+y P^{\perp}, \quad k_{1}^{+}=(1-y) P^{+}, k_{1}^{\perp}=-\kappa_{2}^{\perp}+(1-y) P^{\perp} .
$$

Then

$$
k^{+}=k_{1}^{+}-q^{+}=(x-y) P^{+}, \quad k^{\perp}=k_{1}^{\perp}-q^{\perp}=\kappa_{1}^{\perp}-\kappa_{2}^{\perp}+(x-y) P^{\perp} .
$$

The energy difference appearing in the two energy denominators are, then,

$$
\begin{aligned}
p_{1}^{-}-k_{1}^{-}-k_{3}^{-} & =-\frac{\left(\kappa_{2}^{\perp}\right)^{2}}{P^{+}}\left(\frac{1}{y}+\frac{1}{1-y}\right) \\
p_{1}^{-}-k_{3}^{-}-k^{-}-q^{-} & =-\frac{1}{P^{+}}\left[\frac{\left(\kappa_{2}^{\perp}\right)^{2}}{y}+\frac{\left(\kappa_{1}^{\perp}\right)^{2}}{1-x}+\frac{\left(\kappa_{1}^{\perp}-\kappa_{2}^{\perp}\right)^{2}}{x-y}\right] .
\end{aligned}
$$

The vertex factors are

$$
\begin{aligned}
& -2 \frac{k_{3}^{\perp}}{k_{3}^{+}}+\sigma^{\perp} \frac{\sigma^{\perp} \cdot k^{\perp}}{k^{+}}+\frac{\sigma^{\perp} \cdot p_{2}^{\perp}}{p_{2}^{+}} \sigma^{\perp}=\frac{1}{P^{+}}\left[-2 \frac{\left(\kappa_{1}^{\perp}-\kappa_{2}^{\perp}\right)}{x-y}-\sigma^{\perp} \cdot \frac{\sigma^{\perp} \cdot \kappa_{2}^{\perp}}{1-y}+\frac{\sigma^{\perp} \cdot \kappa_{1}^{\perp}}{x} \sigma^{\perp}\right], \\
& -2 \frac{q^{\perp}}{q^{+}}+\sigma^{\perp} \frac{\sigma^{\perp} \cdot k_{1}^{\perp}}{k_{1}^{+}}+\frac{\sigma^{\perp} \cdot k^{\perp}}{k^{+}} \sigma^{\perp}=\frac{1}{P^{+}}\left[2 \frac{\kappa_{1}^{\perp}}{1-x}+\sigma^{\perp} \frac{\sigma^{\perp} \cdot \kappa_{2}^{\perp}}{y}-\frac{\sigma^{\perp} \cdot \kappa_{2}^{\perp}}{1-y} \sigma^{\perp}\right] \\
& -2 \frac{k_{3}^{\perp}}{k_{3}^{+}}+\sigma^{\perp} \frac{\sigma^{\perp} \cdot p_{1}^{\perp}}{p_{1}^{+}}+\frac{\sigma^{\perp} \cdot k_{1}^{\perp}}{k_{1}^{+}} \sigma^{\perp}=\frac{1}{P^{+}}\left[-2 \frac{\left(\kappa_{1}^{\perp}-\kappa_{2}^{\perp}\right)}{x-y}+\frac{\sigma^{\perp} \cdot \kappa_{2}^{\perp}}{y} \sigma^{\perp}\right] .
\end{aligned}
$$

Thus the vertices and energy denominators appearing in Fig. 2(a) are manifestly invariant under the Galilean boosts in the transverse plane and this is a general property of any $x^{+}$ ordered diagram in light-front perturbation theory. 


\section{REFERENCES}

[1] K.G. Wilson, T.S. Walhout, A. Harindranath, W.-M. Zhang, R.J. Perry, and St. D. Głazek, Phys. Rev. D 49, 6720, (1994).

[2] S.J. Brodsky, H.-C. Pauli, and S.S. Pinsky, Quantum Chromodynamics and other field theories on the light cone, SLAC-PUB-7484, hep-ph/9705477.

[3] L. Susskind, Phys. Rev. 165, 1535 (1968); D.E. Soper, Ph. D. Thesis, SLAC Report No. 137 (1971); J.B. Kogut and L. Susskind, Phys. Rep. C 8, 75 (1973).

[4] A. Harindranath, in Light-Front Quantization and Non-Perturbative QCD, edited by J.P. Vary and F. Woelz, (International Institute of Theoretical and Applied Physics, Ames, Iowa, 1997), hep-ph/9612244.

[5] J.D. Bjorken, J.B. Kogut, and D.E. Soper, Phys. Rev. D 3, 1382 (1971).

[6] W.-M. Zhang and A. Harindranath, Phys. Rev. D 48, 4881 (1993).

[7] A. Harindranath and Wei-Min Zhang, Phys. Lett. B 390, 359 (1997); A. Harindranath and Wei-Min Zhang, Phys. Lett. B 408, 347 (1997); A. Harindranath, Rajen Kundu, Asmita Mukherjee and James P. Vary, to appear in Physics Letters B, hep-ph/9711298.

[8] R.J. Perry, Phys. Lett. B 300, 8 (1993).

[9] S.J. Brodsky and G.P. Lepage, in Perturbative Quantum Chromodynamics, edited by A.H. Mueller (World Scientific, Singapore, 1988), and references therein.

[10] A. Harindranath and W.-M. Zhang, Phys. Rev. D 48, 4903 (1993).

[11] See, for example, R.J. Perry, Ann. Phys. 232, 116 (1994).

[12] J. D. Bjorken and S. D. Drell, Relativistic Quantum Fields, (McGraw-Hill, 1965).

[13] For an overview of this approach, see A. Harindranath and Rajen Kundu, preprint (1996) hep-ph/9606433; A. Harindranath, Rajen Kundu, and Wei Min Zhang, in preparation. 


\section{List of Figures}

1. The canonical quark-gluon vertex in light-front QCD.

2. Contribution to quark-gluon vertex from contributions involving two quark-gluon vertices.

3. Contribution to quark-gluon vertex from contributions involving one quark-gluon vertex and one three gluon vertex.

4. Contribution to quark-gluon vertex from fermion wavefunction renormalization.

5. Contribution to quark-gluon vertex from gluon wavefunction renormalization. 


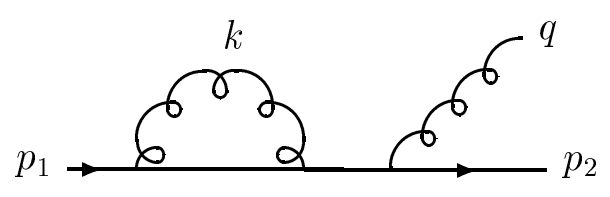

(a)

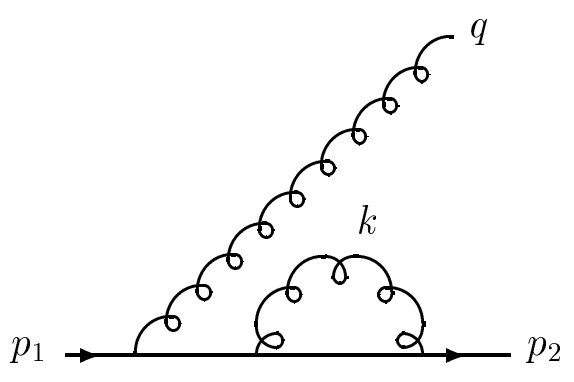

(b)

Figure 4

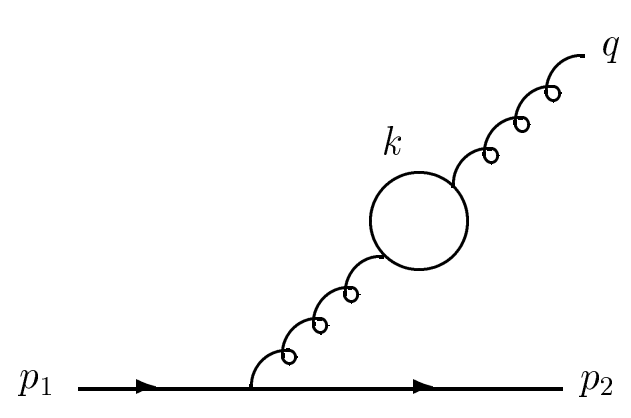

(a)

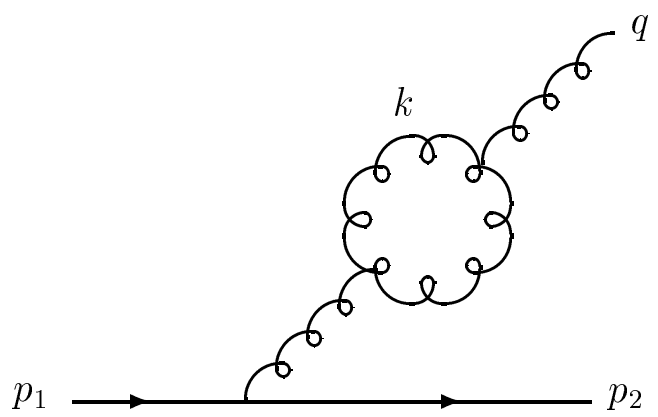

(b)

Figure 5 


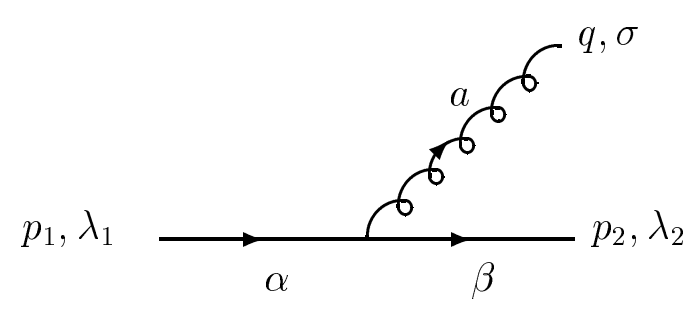

Figure 1

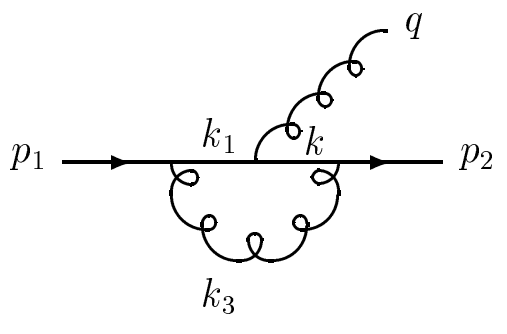

(a)

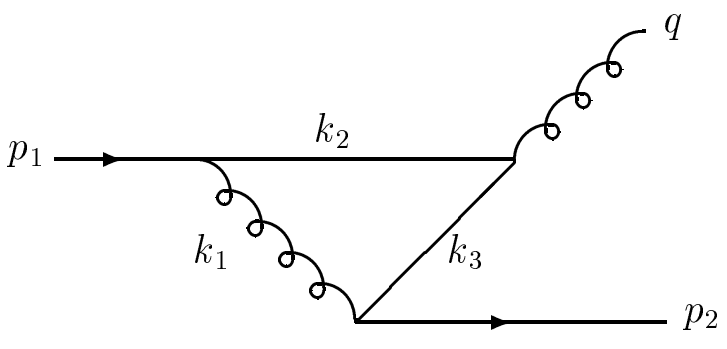

(b)

Figure 2

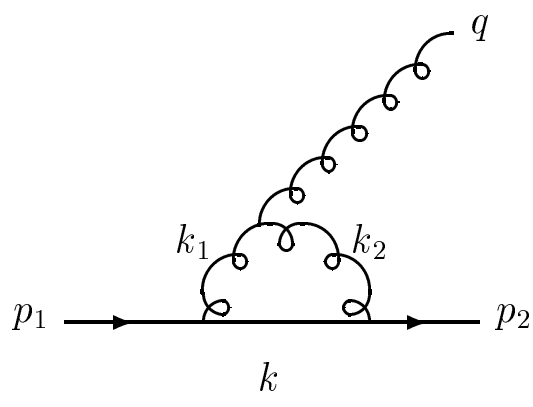

(a)

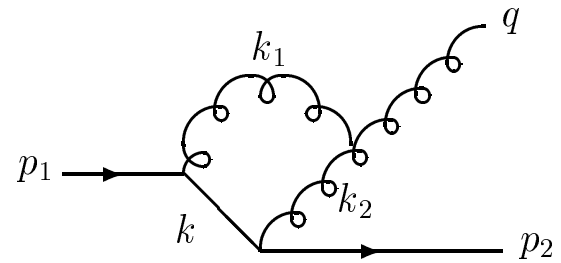

(b)

Figure 3 